\title{
Thin current sheets caused by plasma flow gradients in space and astrophysical plasma
}

\author{
D. H. Nickeler ${ }^{1,2}$ and T. Wiegelmann ${ }^{2}$ \\ ${ }^{1}$ Astronomical Institute AV ČR Ondřejov, Fričova 298, 25165 Ondrejov, Czech Republic \\ ${ }^{2}$ Max-Planck-Institut für Sonnensystemforschung, Max-Planck-Strasse 2, 37191 Katlenburg-Lindau, Germany \\ Received: 20 May 2010 - Revised: 3 August 2010 - Accepted: 5 August 2010 - Published: 13 August 2010
}

\begin{abstract}
Strong gradients in plasma flows play a major role in space and astrophysical plasmas. A typical situation is that a static plasma equilibrium is surrounded by a plasma flow, which can lead to strong plasma flow gradients at the separatrices between field lines with different magnetic topologies, e.g., planetary magnetospheres, helmet streamers in the solar corona, or at the boundary between the heliosphere and interstellar medium. Within this work we make a first step to understand the influence of these flows towards the occurrence of current sheets in a stationary state situation. We concentrate here on incompressible plasma flows and 2-D equilibria, which allow us to find analytic solutions of the stationary magnetohydrodynamics equations (SMHD). First we solve the magnetohydrostatic (MHS) equations with the help of a Grad-Shafranov equation and then we transform these static equilibria into a stationary state with plasma flow. We are in particular interested to study SMHD-equilibria with strong plasma flow gradients perpendicular to separatrices. We find that induced thin current sheets occur naturally in such situations. The strength of the induced currents depend on the Alfvén Mach number and its gradient, and on the magnetic field.
\end{abstract}

Keywords. Magnetospheric physics (Magnetopause, cusp, and boundary layers) - Solar physics, astrophysics, and astronomy (Corona and transition region) - Space plasma physics (Kinetic and MHD theory)

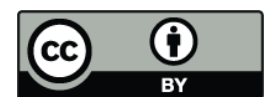

Correspondence to: D. H. Nickeler (nickeler@asu.cas.cz)

\section{Introduction}

Plasma flows around separatrices play an important role in many astrophysical and space plasmas. Significant flows occur thereby mainly on open field lines, while the plasma on closed field lines is approximately at rest. One example is the magnetosphere surrounded by the solar wind flow, where both regions are separated by the magnetopause. Another example are coronal helmet streamers, where the closed arcade type magnetic structures are surrounded by open magnetic field lines on which the stationary solar wind is streaming.

A third example, but with different constraints, is the region far away from a star, which is embedded in the counterflowing interstellar medium. Due to the interaction between the stellar wind, e.g., the solar wind, and the counterflowing interstellar medium, a separatrix forms, separating the "inner" stellar wind from the "outer" interstellar medium: the domain inside is called an astrosphere (heliosphere for the sun), the corresponding separatrix is called an astropause (heliopause). This is a similar situation as described for the helmet streamers in the paragraph before, namely different flow regimes inside and outside of some boundary layer ("inner" and "outer" field lines). In the case of astrospheres/of the heliosphere, however, the scenario implies a structure with almost completely open field lines. Additionally, the flow is non-zero also on the "inner" field lines, but shows a strong gradient (see, e.g., Baranov et al., 1970; Baranov and Krasnobaev, 1971; Nickeler et al., 2006, for details).

These situations where regions with and without plasma flow are separated by rather thin boundary layers necessarily lead to strong flow gradients in these layers. Within this work we aim to study the relation of these flow gradients to current sheets. Thin current sheets are important, because due to current driven micro-instabilities a fine resistivity occurs in these regions and the usual assumption of an ideal conducting

Published by Copernicus Publications on behalf of the European Geosciences Union. 
space plasma breaks down. Consequently resistive plasma instabilities like magnetic reconnection can occur as a consequence of current sheets and when additional sufficient free energy for eruptions is available in the configuration (see for example Schindler and Birn, 1993). Such processes occur, e.g., as substorms in magnetospheres and flares in the solar corona (see Birn and Hesse, 2009, for a recent study of similarities and difference between substorms and flares). Wiegelmann and Schindler (1995), and Becker et al. (2001) studied the formation of thin current sheets as a sequence of quasi-static magnetotail equilibria. Quasi-static means that dynamical effects and the influence of plasma flow can be neglected compared to magnetic forces and pressure gradients. This assumption is well fulfilled in the magnetosphere during quiet times (Schindler and Birn, 1982) and the strongest currents form in the center of the magnetospheric plasma sheet. The influence of a field-aligned parallel plasma flow in magnetospheres has been studied for example in Birn (1991) and for solar MHD-equilibria in Wiegelmann et al. (1998), Petrie and Neukirch (1999), Petrie et al. (2002), and Petrie et al. (2005). A significant influence of a smooth plasma flow itself onto the magnetic field and plasma configuration is rather low for flow speed well below the Alfvén speed $v \ll v_{\mathrm{A}}$. Far less studied has been the influence of significant small scale gradients in the plasma flow, which is the topic of this paper. We are in particular interested to investigate to which extend thin current sheets at boundary layers can be associated with corresponding gradients in the plasma flow. We outline the paper as follows. In Sect. 2 we present the basic equations, outline how static equilibria can be transformed into stationary ones, and discuss the relation between sharp flow gradients and the occurrence of thin current sheets. Sections 3 and 4 contain applications to solar coronal and magnetospheric structures, respectively. Finally we summarize our results in Sect. 5.

\section{Basic assumptions and equations}

We apply the theory of ideal MHD. This is justified, because of the high conductivity in many space and astrophysical plasmas. For simplicity and to concentrate on essential "flow" effects, we restrict our research to configurations with an incompressible plasma flow. We are interested in the physical effects that occur for large gradients of the Alfvén Mach number perpendicular to the field lines. We are aware that compressible effects might become important for some space plasma applications, in particular for stratified plasmas like the solar corona and chromosphere, especially on large scales, see, e.g. Petrie et al. (2002) or Petrie et al. (2005).

For stationary, ideal and incompressible MHD we have to solve the following equations: mass continuity Eq. (1), the Euler or momentum equation with isotropic pressure $P$ (Eq. 2), the stationary induction equation including the ideal Ohm's law (Eq. 3), Ampère's law (Eq. 4), the solenoidal con- dition for the magnetic field (Eq. 5), and the condition for incompressibility (Eq. 6):

$$
\begin{aligned}
\nabla \cdot(\rho \mathbf{v}) & =0, \\
\rho(\mathbf{v} \cdot \nabla) \mathbf{v} & =\boldsymbol{j} \times \boldsymbol{B}-\nabla P, \\
\nabla \times(\mathbf{v} \times \boldsymbol{B}) & =\mathbf{0}, \\
\nabla \times \boldsymbol{B} & =\mu_{0} \boldsymbol{j}, \\
\nabla \cdot \boldsymbol{B} & =0, \\
\nabla \cdot \mathbf{v} & =0,
\end{aligned}
$$

where $\rho$ is the mass density, $\boldsymbol{v}$ the plasma velocity, $\boldsymbol{B}$ the magnetic field, $\boldsymbol{j}$ the current density vector, and $P$ the thermal or plasma pressure.

Due to the incompressibility, the mass continuity equation can be written as $\boldsymbol{v} \cdot \boldsymbol{\nabla} \rho=0$, so that the density is constant on streamlines. We now introduce the auxilliary flow vector or streaming vector $\boldsymbol{w}:=\sqrt{\rho} \mathbf{v}$. With the Bernoulli pressure, defined by $\Pi:=P+\frac{1}{2} \boldsymbol{w}^{2}$, we can rewrite the above equations as

$$
\begin{aligned}
\nabla \cdot \boldsymbol{w} & =0, \\
\frac{1}{\mu_{0}}(\boldsymbol{\nabla} \times \boldsymbol{B}) \times \boldsymbol{B}-(\boldsymbol{\nabla} \times \boldsymbol{w}) \times \boldsymbol{w} & =\nabla \Pi, \\
\nabla \times\left(\frac{1}{\sqrt{\rho}} \boldsymbol{w} \times \boldsymbol{B}\right) & =\mathbf{0}, \\
\nabla \cdot \boldsymbol{B} & =0 .
\end{aligned}
$$

The momentum equation Eq. (8) is written such that the analogy with magnetohydrostatic equilibria (MHS), given by

$\mu_{0}^{-1}(\nabla \times \boldsymbol{B}) \times \boldsymbol{B}=\nabla P$,

is obvious.

The assumption of a field-aligned flow enhances the probability that the flow is stable in the frame of ideal MHD, (see the discussion in Hameiri, 1998). The assumption of $\boldsymbol{v} \times \boldsymbol{B}=$ 0 leads to a vanishing electric field in ideal MHD. This can be seen with the help of the uncurled induction equation, Eq. (3), which is basically ideal Ohm's law $\boldsymbol{E}+\boldsymbol{v} \times \boldsymbol{B}=\mathbf{0}$ $(\boldsymbol{E}=$ electric field). Therefore the stationary ideal Ohm's law (Eq. 9) is fulfilled identically. Under these assumptions the set of equations reduces to:

$$
\begin{aligned}
& \boldsymbol{B} \cdot \nabla M_{\mathrm{A}}=0, \\
& \nabla \Pi=\frac{\left(1-M_{\mathrm{A}}^{2}\right)(\nabla \times \boldsymbol{B}) \times \boldsymbol{B}}{\mu_{0}}-\frac{|\boldsymbol{B}|^{2}}{2 \mu_{0}} \nabla\left(1-M_{\mathrm{A}}^{2}\right), \\
& \nabla \cdot \boldsymbol{B}=0,
\end{aligned}
$$

where $M_{\mathrm{A}}$ is the Alfvén Mach number, defined via

$\boldsymbol{w} \equiv \pm M_{\mathrm{A}} \boldsymbol{B} / \sqrt{\mu_{0}}$,

and where the \pm indicates that if the pair $(\boldsymbol{w}, \boldsymbol{B})$ is a solution of the Eqs. (8) and (13), then $(-\boldsymbol{w}, \boldsymbol{B})$ is also a solution, and basically also $(\boldsymbol{w},-\boldsymbol{B})$ and $(-\boldsymbol{w},-\boldsymbol{B})$. We will mainly parameterize this behaviour by $M_{\mathrm{A}}$ and by $\boldsymbol{B}$, starting from 
the viewpoint of MHS theory, as the form (13) of the incompressible SMHD equations allows to derive transformation equations which transform MHS equilibria into stationary ones (see Gebhardt and Kiessling, 1992; Nickeler et al., 2006, for the mathematical details).

\section{$2.1 \quad$ 2-D stationary states}

The theory explained so far is general. In the following we concentrate on configurations with one axis of symmetry, e.g. the z-axis in a cartesian coordinate system $x, y, z$. Consequently all quantities are functions of $x$ and $y$ only and we can solve the solenoidal Eq. (14) by introducing a flux function $\alpha(x, y)$ of the form $\boldsymbol{B}=\nabla \alpha \times \boldsymbol{e}_{z}$. This reduces the stationary incompressible equations in 2-D to

$M_{\mathrm{A}}=M_{\mathrm{A}}(\alpha)$,

$\nabla \Pi=-\frac{\left(1-M_{\mathrm{A}}^{2}\right) \Delta \alpha \nabla \alpha}{\mu_{0}}-\frac{|\nabla \alpha|^{2}}{2 \mu_{0}} \nabla\left(1-M_{\mathrm{A}}^{2}\right)$.

Now we perform a transformation by assuming that $\alpha(x, y)$ is a function of another "flux function" $A(x, y)$, i.e. $\alpha=\alpha(A)$, such that the stationary equation, Eq. (17), reduces to a form of the equation mathematically similar to the GradShafranov equation

$$
\frac{d P_{\mathrm{MHS}}}{d A}=-\frac{1}{\mu_{0}} \Delta A
$$

describing MHS equilibria.

Then the equivalence between the MHS equation and the momentum equation of ideal, stationary but non-static MHD with incompressible, field-aligned flow is shown. As $\alpha$ is a function of $A, M_{\mathrm{A}}$ is also a function of the "new" flux function $A$. Derivatives with respect to $A$ will now and in the following be expressed by a prime, e.g., $d \alpha / d A=\alpha^{\prime}$. With the help of the relation $M_{\mathrm{A}}=M_{\mathrm{A}}(A)$ we can rewrite the Euler equation Eq. (17)

$$
\begin{aligned}
\nabla \Pi= & -\frac{\left(1-M_{\mathrm{A}}^{2}\right)\left(\alpha^{\prime \prime}(\nabla A)^{2}+\alpha^{\prime} \Delta A\right) \alpha^{\prime} \nabla A}{\mu_{0}} \\
& -\frac{\alpha^{\prime 2}|\nabla A|^{2}}{2 \mu_{0}} \nabla\left(1-M_{\mathrm{A}}^{2}\right) \\
= & -\frac{\left(1-M_{\mathrm{A}}^{2}\right)}{\mu_{0}} \alpha^{\prime 2} \Delta A \nabla A \\
& -\frac{(\nabla A)^{2} \nabla A}{2 \mu_{0}}\left[\left(1-M_{\mathrm{A}}^{2}\right) \alpha^{\prime 2}\right]^{\prime} .
\end{aligned}
$$

Let us remark that the Alfvén Mach number $M_{\mathrm{A}}$ can be expressed as a function of $\alpha$ or $A$, but is not restricted further. We have therefore the freedom to choose this function arbitrary without loss of generality. A reasonable choice to eliminate the term $(\nabla A)^{2}$ in Eq. (19) is

$$
\left(1-M_{\mathrm{A}}^{2}\right) \alpha^{\prime 2} \equiv 1,
$$

and therefore the Euler Eq. (19) simplifies to a single partial differential equation for the new flux function $A(x, y)$

$\nabla \Pi=-\frac{1}{\mu_{0}} \Delta A \nabla A \Rightarrow \frac{d \Pi}{d A}=-\frac{1}{\mu_{0}} \Delta A$.

In any case, Eq. (21) is mathematically identical with Eq. (18), but contains plasma flow. Physically this equation reduces to the static Grad-Shafranov equation only for the limit $M_{\mathrm{A}} \rightarrow 0$, implying $\Pi(A) \rightarrow P(A)$. Consequently, any solution $A(x, y)$ of the "MHS" Eq. (21) (or equivalently the MHS Eq. 18) can be used to derive a solution of the stationary, incompressible MHD by integrating Eq. (20)

$\alpha= \pm \int \frac{d A}{\sqrt{1-M_{\mathrm{A}}(A)^{2}}}$.

With this form we can specify a plasma flow via the Alfvén Mach number $M_{\mathrm{A}}(A)$. Because $A$ is constant on magnetic field lines this is also true for $M_{\mathrm{A}}$ and $\alpha$. Physically this means that we can specify on which field lines plasma is flowing with a certain Mach number. It is in particular possible to calculate separatrix field lines in the static case and specify plasma flow only on one side of this separatrix, e.g. to model plasma flow around a static magnetosphere or helmet streamer configuration.

Equation (22) is also equivalent to

$A= \pm \int \sqrt{1-M_{\mathrm{A}}(\alpha)^{2}} d \alpha$.

Some care has to be taken for multi-valued functions $M_{\mathrm{A}}(A)$ or $M_{\mathrm{A}}(\alpha)$, where one has to distinguish between the different branches of solutions. This is, however, not a major problem and similar to the problem of multi-valued functions $\Pi(A)$ in the static Grad-Shafranov theory, which has been addressed in Wiegelmann et al. (1998) to model triple coronal helmet streamer configurations.

\subsubsection{Influence on the electric current density}

Inserting the ansatz $\boldsymbol{B}=\nabla \alpha \times \boldsymbol{e}_{z}$ into Eq. (4) and by computing the Laplacian of $\alpha$ with Eq. (22) we find the connection between the Alfvén Mach number and current density

$$
\begin{aligned}
-\mu_{0} j_{z} & =\Delta \alpha \\
& = \pm \frac{M_{\mathrm{A}} M_{\mathrm{A}}^{\prime}}{\left(1-M_{\mathrm{A}}^{2}\right)^{\frac{3}{2}}}(\nabla A)^{2} \pm \frac{1}{\sqrt{1-M_{\mathrm{A}}^{2}}} \Delta A .
\end{aligned}
$$

The \pm sign again reflects the freedom of the transformation relations Eqs. (22) and (23) with respect to the direction of the magnetic field and the symmetry of the Lorentz force. The first term in Eq. (24) corresponds to a current induced by the plasma flow and the second part modifies (enhances) the static equilibrium current $-\mu_{0} j_{z \text {,static }}=-\Delta A$.

For static potential fields this part of the current vanishes also in the stationary state with flow, i.e. as $\Delta A=0$ only the first term on the right side of Eq. (24) contributes to the 

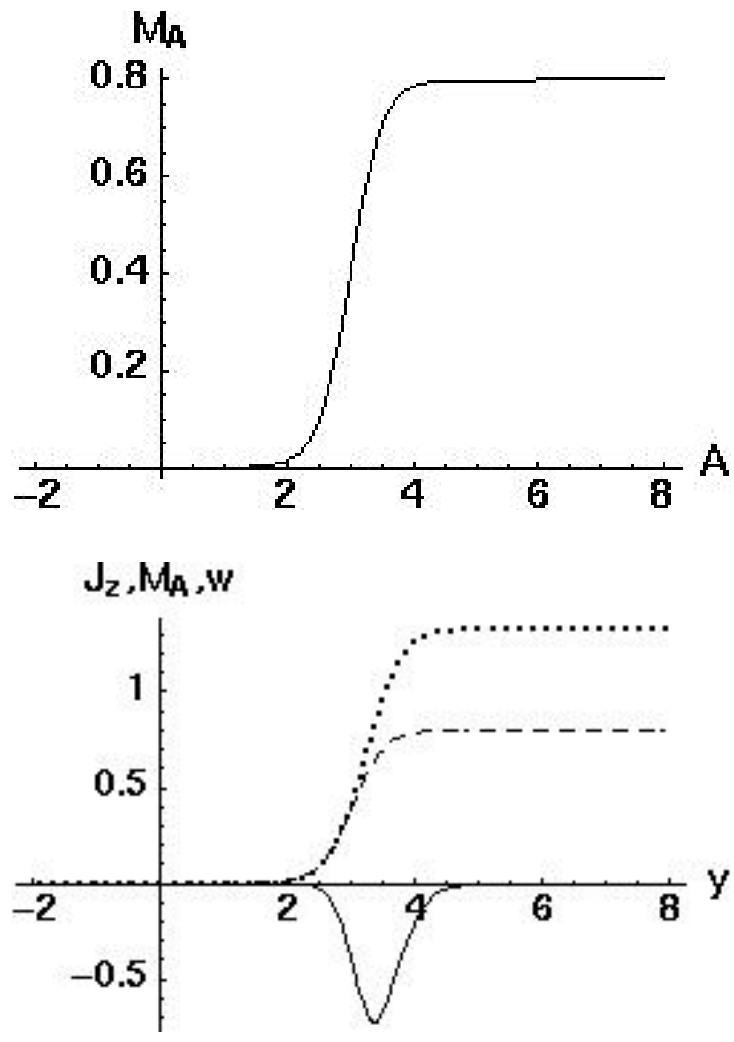

Fig. 1. Transformation from a one-dimensional homogenous current-free potential field $A=B_{0} y$. Top panel: $M_{\mathrm{A}}(A)$ as defined in (29), with $M_{1}=0.4, M_{2}=0.8, d=2, A_{c}=3$ Bottom panel: $M_{\mathrm{A}}(y)$ in dashed line, $w(y)$ in thick dotted line and the resulting electric current density $j_{z}(y)$ in solid line.

electric current density. We expand Eq. (24) for small Alfvén Mach numbers $M_{\mathrm{A}} \ll 1$ which leads to

$-\mu_{0} j_{z}= \pm\left(M_{\mathrm{A}}+\frac{3}{2} M_{\mathrm{A}}^{3}\right) M_{\mathrm{A}}^{\prime}(\nabla A)^{2} \pm\left(1+\frac{1}{2} M_{\mathrm{A}}^{2}\right) \Delta A$,

and if we neglect all quadratic and higher terms in $M_{\mathrm{A}}$, we find:

$-\mu_{0} j_{z}= \pm M_{\mathrm{A}} M_{\mathrm{A}}^{\prime}(\nabla A)^{2} \pm \Delta A$.

Inserting the definitions of the magnetic field and equilibrium current and using the definition $M_{\mathrm{A}}^{\prime} \nabla A=\frac{d M_{\mathrm{A}}}{d A} \nabla A=\nabla M_{\mathrm{A}}$ (this can be done also already in Eq. (24) and does not depend on the assumption of small Mach numbers), we get from Eq. (26)

$-\mu_{0} j_{z}= \pm\left(M_{\mathrm{A}} \nabla M_{\mathrm{A}} \cdot \nabla A+j_{z, \text { static }}\right)$.

Consequently for small Alfvén Mach numbers the equilibrium currents are basically unmodified by the plasma flow and the induced currents depend linearly on the magnetic field strength, the Alfvén Mach number and the gradient of the Alfvén Mach number. For further approximations on the relative strength of the equilibrium currents and the flow induced currents we assume that the magnetic field equilibrium does change on a length scale $l_{\text {static }}$ and the plasma flow on a scale $l_{\text {flow }}$, which allows us to roughly approximate the gradient and Laplacian:

$$
-\mu_{0} j_{z} \approx \pm\left(M_{\mathrm{A}} \frac{M_{\mathrm{A}}}{l_{\text {flow }}} B+\frac{B}{l_{\text {static }}}\right) \text {. }
$$

So to compare the relative strength of the two contributions we have to compare $\frac{M_{\mathrm{A}}^{2}}{l_{\text {flow }}}$ and $\frac{1}{l_{\text {static }}}$ and get as the ratio of induced and equilibrium current $M_{\mathrm{A}}^{2} \frac{l_{\text {static }}}{l_{\text {flow }}}$. Consequently we get (for slow plasma flows with $M_{\mathrm{A}} \ll 1$ ) only a significantly large induced current if the plasma flow changes on a much smaller length scale as the typical scale of the configuration $l_{\text {flow }} \ll l_{\text {static }}$. Such a situation is typically fulfilled at boundary layers, e.g., the magnetopause or the separatrix between open and closed field lines in coronal helmet streamers.

\section{Application to coronal helmet streamers and plasmoids}

In the following we provide some example solutions for MHD-equilibria with plasma flow. We construct these configurations by first solving the MHS problem (Eq. 21) and then by transforming the resulting static flux function $A(x, y)$ into the solution of the stationary problem $\alpha(x, y)$ with the help of Eq. (22). We prescribe the Alfvén Mach number as a function of $A$ in the useful form

$M_{\mathrm{A}}(A)=M_{1}+\left(M_{2}-M_{1}\right) \tanh \left(d\left(A-A_{c}\right)\right)$,

where $M_{1}, M_{2}, d$, and $A_{c}$ are free parameters ${ }^{1}$, the scale on which the flow changes $\left(d \propto 1 / l_{\text {flow }}\right.$ is an inverse length), and the value of the separatrix field line $A_{c}$. The functional form of $M_{\mathrm{A}}$ in Eq. (29) has been chosen in order to provide the strongest flow gradient at the separatrix field line $A_{c}$. Figure 1 top panel shows $M_{\mathrm{A}}$ as a function of $A$ for $M_{1}=0.4$, $M_{2}=0.8, d=2$, and $A_{c}=3$.

As an example we apply the transformation (22) with $M_{\mathrm{A}}$ in the form of Eq. (29) to a homogeneous potential magnetic field $\boldsymbol{B}=B_{0} \boldsymbol{e}_{x}$ with $B_{0}=1$. This is a simple 1-D equilibrium with the static flux function $A=B_{0} y$ and all quantities (both in the static and stationary case) are only a function of $y$. Figure 1 bottom panel shows the Alfvén Mach number $M_{\mathrm{A}}(y)$ (dashed line), the streaming vector $w(y)$ (dotted line) and the corresponding formation of a current sheet $j_{z}(y)$ at the separatrix field line $A_{c}=3$ (solid line).

In the following we study more sophisticated static equilibria and their transformation to stationary incompressible MHD-equilibria. For a better visualization we will present examples of MHS equilibria that do not show the extremely small scale flows compared to the equilibrium current scales. We therefore have to use larger Alfvén Mach numbers.

\footnotetext{
${ }^{1}$ Please note that the Alfvén Mach number can become negative for plasma flows antiparallel to the field lines.
} 


\subsection{Linear MHS equilibria}

To derive 2-D static equilibria we solve the Grad-Shafranov Eq. (21) for a linear current. Such configurations have been studied for triple coronal helmet streamer configurations in Wiegelmann (1998), but here we limit our research to single helmet streamers and concentrate on the effect of plasma flow on open field lines. A linear current means that the function $\Pi(A)$ in Eq. (21) has the form $\Pi(A)=\frac{c^{2}}{2} A^{2}$. In this case the Grad-Shafranov equation reduces to a linear Helmholtz equation

$$
-\Delta A=c^{2} A
$$

and can be solved by separation of variables. Let us remark that in general the static Grad-Shafranov equation has the form $\Pi(A)=p(A)+\frac{B_{z}^{2}}{2}$, with the plasma pressure $p$ and a magnetic shear field $B_{z}$ in the invariant direction. In the case of $p(A)=0$ one obtains linear force-free configurations and else static equilibria. In both cases the electric current $j_{z}(A)=\frac{\partial \Pi}{\partial A}$ is linear in $A$. The particular choice $c=0$ corresponds to current-free potential fields. As solution of Eq. (30) one gets by separation of variables

$$
\begin{aligned}
& A(x, y)=B_{0} \exp \left(-\frac{v \pi y}{L}\right) \cos \left(\frac{k \pi x}{L}\right) \text { for } c<k, \\
& A(x, y)=B_{0} \cos \left(\frac{\omega \pi y}{L}\right) \cos \left(\frac{k \pi x}{L}\right) \text { for } c>k,
\end{aligned}
$$

with $v=\sqrt{k^{2}-c^{2}}$ and $\omega=\sqrt{c^{2}-k^{2}}$. Linear combinations of these particular solutions are also solutions of the linear Helmholtz-equation (Eq. 30). These solutions were also studied by Hood and Anzer (1990) modeling prominence arcades and Petrie (2006) modeling coronal loops. Here we consider only three particular cases with $B_{0}=k=L=1$ and different values of $c$. The top panels of Figs. 2, 3, and 4 show magnetic field lines (equi-contour plots of the flux function $A(x, y)$ for $c=0, c=0.9$, and $c=1.2$, respectively). The case $c=0$ in Fig. 2 corresponds to a current-free potential field. Introducing a moderate linear current with $c<k$ leads to a stretching of the configuration (top panel in Fig. 3) and a smooth electric current density distribution (second panel in Fig. 3). For the case $c>k$, as shown in the top panel of Fig. 4, the magnetic topology changes and wet get plasmoidlike configurations, which, however, also have a smooth current density distribution in equilibrium (second panel).

\subsubsection{Transformation to stationary states}

We transform these static equilibria into stationary ones using Eq. (22) and $M_{\mathrm{A}}$ in the form (29) with $d=5, M_{1}=0.0$, $M_{2}=0.8, A_{c}=0$. We choose $M_{2}>M_{1}$, in order to prescribe a plasma flow on the outside $(x= \pm 0.5)$ of the configuration, where the flux function becomes negative. Inside the streamer and plasmoid $(-0.5<x<0.5)$ the flux function is positive. The plasma flow is chosen in order to be
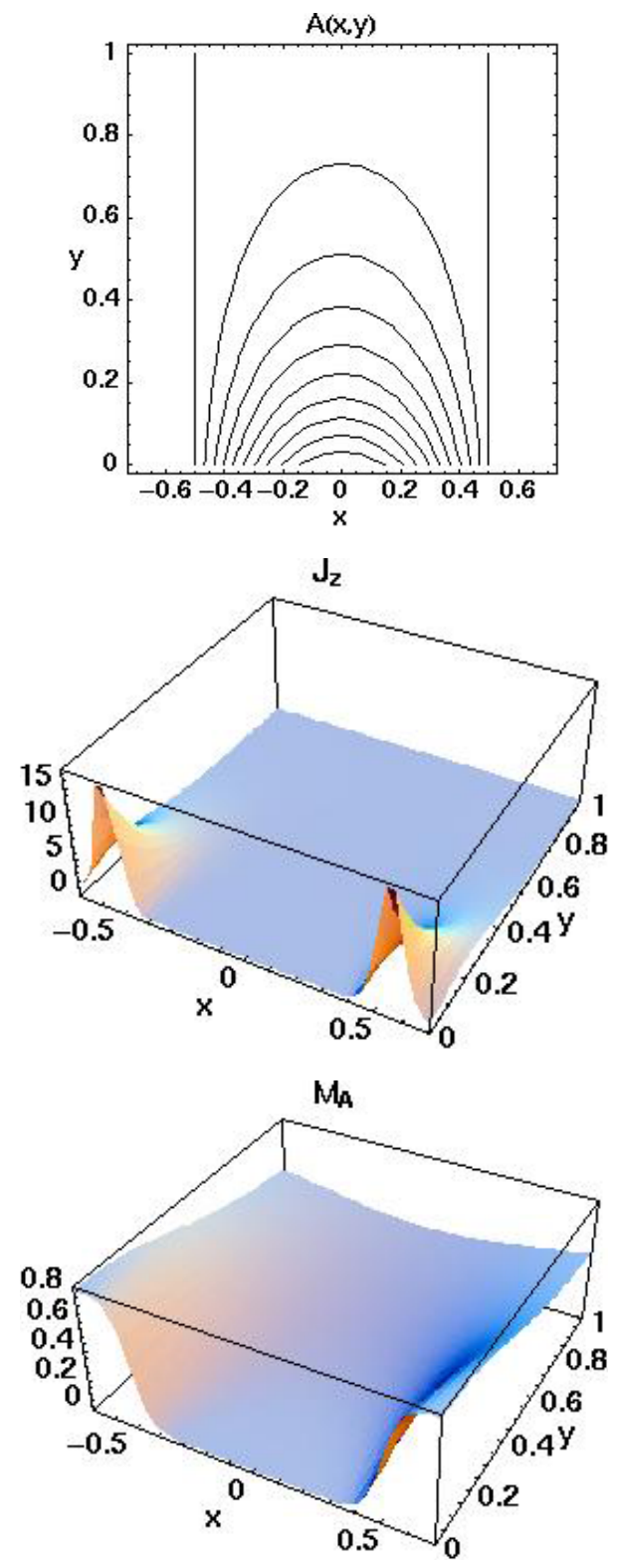

Fig. 2. 2-D potential field configurations in the form $A(x, y)=$ $B_{0} \exp (-k \pi y / L) \cos \left(-\sqrt{k^{2}-c^{2}} \pi x / L\right)$, with $B_{0}=k=L=1, c=$ 0.0 and the Alfvén Mach number profile function (29) with $d=5$, $M_{1}=0.4, M_{2}=0.0, A_{c}=0$. This profile has a steep gradient at the boundary between open and closed field lines. Top panel: magnetic field lines (contour lines of $A(x, y)$ ), center: formation of flow driven current sheets $J_{z}(x, y)$, Bottom: Alfvén Mach number $M_{\mathrm{A}}(x, y)$.

maximal at the open separatrix field line and the plasma is basically at rest inside the configuration, where the magnetic field lines are closed (bottom panels in Figs. 2, 3, 4). With the current transformation Eq. (24) we compute the total current density: In the case of the static potential field currents only occur from the first term in Eq. (24) and are driven by 

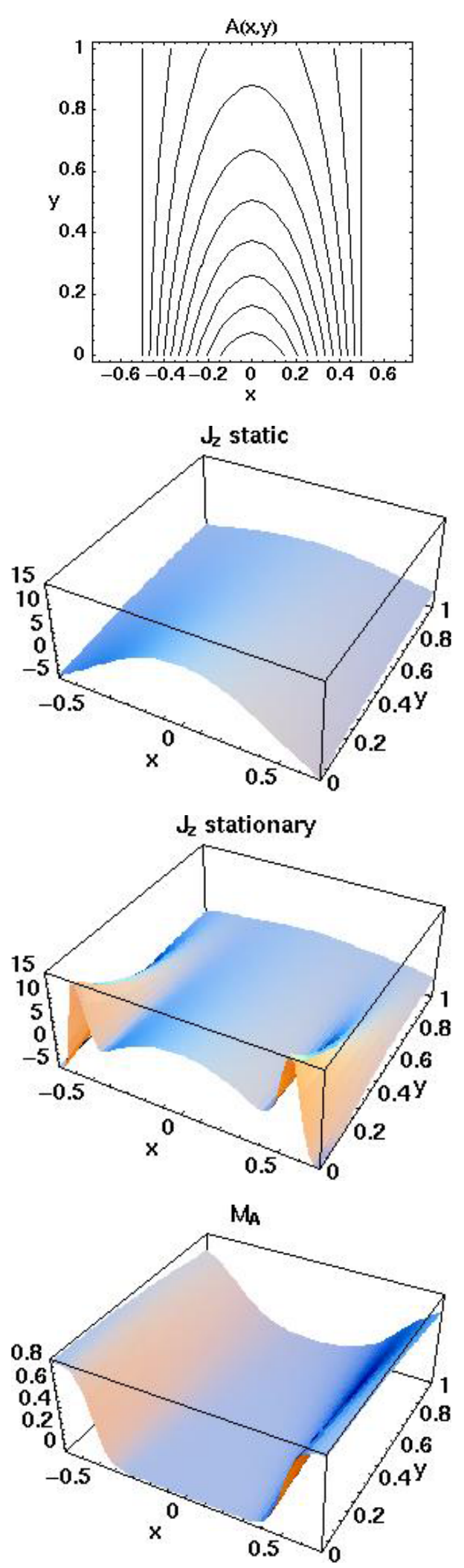

Fig. 3. Same transformation as in Fig. 2, including a linear MHS current $J_{z}(A)=c \cdot A, c=0.9$. Second and third panel show the split of linear (MHS, without flow) and the full current (with flow).
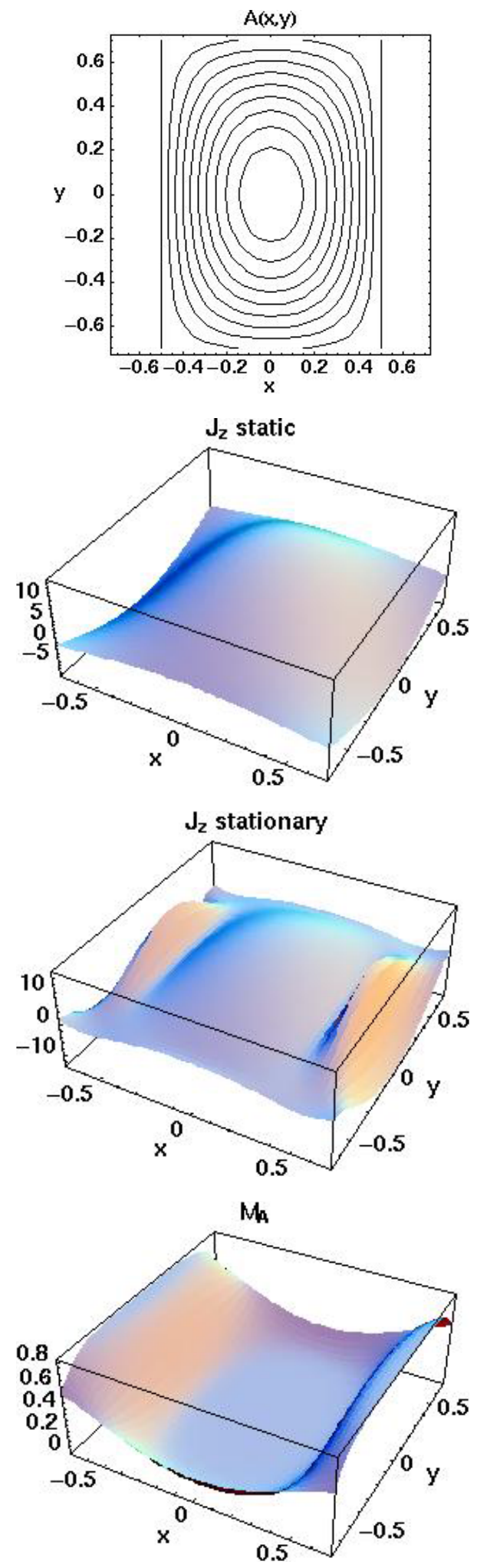

Fig. 4. Same transformation as in Fig. 2, but including a linear MHS current in the form $J_{z}(A)=c \cdot A$ with $c=1.2$. Here $c>k$. 
the gradient of the plasma flow (center panel in Fig. 2). As a consequence of the sharp gradient in the flow, a thin current sheet forms at the separatrix $(x= \pm 0.5)$. Such a thin current sheet forms also additional to the smooth equilibrium currents for the linear current cases (third panels in Figs. 3 and 4). In regions with weak or no plasma flow the equilibrium current does basically not change, whereas a current sheet forms at the separatrix field line. Due to the strong gradient in the plasma flow this induced current sheet is much thinner and the current density is higher than in the equilibrium current. The equilibrium currents are strongest in the center of the configuration.

\section{Application to magnetospheres}

\subsection{Non-linear Grad-Shafranov equation, Liouville equation}

In the static case without magnetic shear field the GradShafranov-equation (21) reduces to

$\Delta A=-\mu_{0} \frac{\partial}{\partial A}(p(A))$,

where $p(A)$ is the plasma pressure. Under the assumption of a local thermodynamical equilibrium the plasma pressure function can be derived from kinetic theory in the form (see Schindler, 2006, for details)

$p(A)=\frac{1}{2} \hat{p} \exp (-2 A / \hat{A})$,

where $\hat{A}$ and $\hat{p}$ are normalization constants. This leads to an equation in the form

$\Delta A=\lambda \exp (-c A)$,

with constants

$\lambda=\mu_{0} \frac{\hat{p}}{\hat{A}}$,

$c=\frac{2}{\hat{A}}$,

and the typical lengthscale $\hat{l}$, and the typical magnetic field $\hat{B}$, defined by

$\lambda c=\left(\frac{2 \hat{p}}{\hat{A}^{2} /\left(\hat{l}^{2} \mu_{0}\right)}\right) \equiv \frac{2}{\hat{l}^{2}}$,

$\hat{B}=\frac{\hat{A}}{\hat{l}}$.

Before we continue to compute analytical and exact 2-D solutions, we first present a well-known case in 1-D, namely the Harris-sheet, to explain how the transformation from MHS to incompressible SMHD works.

\subsection{1-D Harris-sheet}

A well known, 1-D equilibrium current sheet solution of the Liouville's Eq. (35) is the Harris-sheet (Harris, 1962). A Harris-sheet like force-free equilibrium has recently been found by Harrison and Neukirch (2009). The Harris sheet is a 1-D-solution, where all quantities depend only on the $y$ coordinate, and is given by

$A(y)=\hat{A} \ln \cosh (y / \hat{l})$.

The static equilibrium quantities for the Harris-sheet are shown with solid lines in Fig. 5. The top panel shows the flux-function $A(y)$, the second panel the corresponding magnetic field $B_{x}$ and the bottom panel the equilibrium electric current density.

\subsubsection{Transformation to stationary states}

We use the transformation Eq. (22) with the Mach number profile (29) and $M_{\max }=0.5, M_{\min }=0, A_{c}=2$, and $d=5$ to derive a stationary equilibrium with plasma flow. The stationary solutions are shown with dashed lines in Fig. 5. The top panel shows the flux function $\alpha(y)$, the second panel the magnetic field $B_{x}$ and the bottom panel the electric current density $j_{z}(y)$ as computed with Eq. (24). Additionally we present the current approximation for $M_{\mathrm{A}} \ll 1$, as computed with Eq. (26) with dotted lines in the bottom panel of Fig. 5. By comparing the static (solid lines) and stationary (dashed) quantities, one can see that both quantities only differ in the region were the flow gradient is high (see third panel). In these regions, where the static equilibrium is separated from the stationary flowing plasma in a thin layer, current sheets form. The spatial scale of these layers is significantly smaller than the typical length scale of the equilibrium current. The electric current density approximation for small Mach number (dotted in bottom panel) shows reasonable agreement with the exact (dashed) solution, even for the not very small maximum Mach number $M_{\mathrm{A}}=0.5$ used here.

\subsection{Exact 2-D magnetospheric equilibrium}

As shown by Liouville (1853), Bandle (1975), Birn et al. (1978) Eq. (35) can be written as:

$4 \frac{\partial^{2} A}{\partial u \partial \bar{u}}=\lambda \exp (-c A)$,

with $u=x+i y$ and $\bar{u}=x-i y$, and this equation has the general solution

$A(u, \bar{u})=\frac{2}{c} \ln \frac{1+\frac{c \lambda}{8}\left|\Psi(u)^{2}\right|}{\left|\frac{\partial \Psi}{\partial u}\right|} \equiv \hat{A} \ln \frac{1+\frac{1}{4 \hat{l}^{2}}\left|\Psi(u)^{2}\right|}{\left|\frac{\partial \Psi}{\partial u}\right|}$.

Every analytic function $\Psi(u)$ generates a solution of Eq. (35). The Liouville equation has also its applications outside plasma physics and solutions in the form of (42) have 

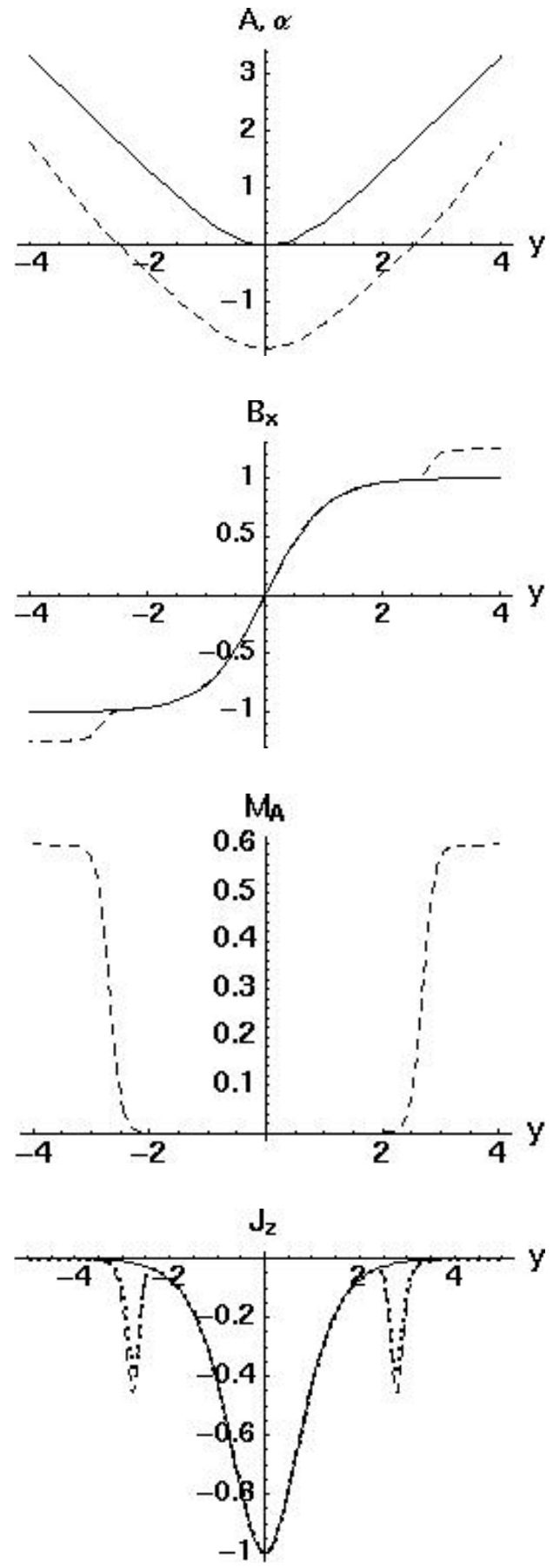

Fig. 5. From top to bottom we show the flux-functions $A(y), \alpha(y)$, the magnetic field $B_{x}(y)$, the Alfvén Mach number $M_{\mathrm{A}}(y)$ and the electric current density $j_{z}(y)$. Solid lines correspond to the magnetostatic case and dashed lines to stationary MHD with the profile (29) and $M_{1}=0, M_{2}=0.5, A_{c}=2$ and $d=5$. In the bottom panel we show additional dotted the approximation for $M_{\mathrm{A}} \ll 1$ as computed with Eq. (27). been used, e.g., by Schmid-Burgk (1967) to investigate a self-gravitating gas layer.

Schindler and Birn (2004) found magnetospheric solutions with the Ansatz

$\Psi(u)=2 \hat{l} \exp \left(i\left(u / \hat{l}+\sqrt{\frac{u / \hat{l}}{\epsilon}}\right)\right)$,

which leads to the solution class of Eq. (42) of the form (see Schindler and Birn, 2004, for details and discussion of the static equilibrium).

$A(x, y) / \hat{A}=\ln \left(\frac{\cosh \left(\frac{y}{\sqrt{2 \epsilon} \sqrt{r+x}}+y\right)}{\sqrt{\frac{1}{r}\left(\frac{1}{4 \epsilon}+\sqrt{\frac{r+x}{2 \epsilon}}\right)+1}}\right)$

with $r=\sqrt{x^{2}+y^{2}}$, where the coordinates here are normalized on $\hat{l}$. For $\epsilon \rightarrow \infty$ the Ansatz for $\Psi$ produces the Harrissheet solution. The top panel of Fig. 6 shows the corresponding magnetic field lines as equi-contour plots of $A(x, y)$ for $\epsilon=1$. For the transformation we used the parameters $M_{1}=0.0, M_{2}=0.95$ and $d=2$ for the Mach number profile in Eq. (29). The second panel contains the equilibrium current density $-j_{z}(x, y)$.

\subsubsection{Transformation to stationary states}

We use the transformation Eq. (22) with the Mach number profile (29) and $M_{\max }=0.95, M_{\min }=0, A_{c}=0$, and $d=2$ to derive a stationary equilibrium with plasma flow. The chosen profile for the flow is smoother, as in previous examples and the maximum Mach number is higher. As a consequence we observe two additional current sheets in the stationary current distribution as computed with Eq. (26) and shown in the third panel of Fig. 6. The thickness of the two current sheets are located in the region with the plasma flow gradient, shown in the bottom panel. The smoother profile of $M_{\mathrm{A}}(A)$ results also in smoother induced current sheets.

\section{Conclusions}

Within this work we studied the relation between plasma flow gradients and current sheets in space plasma. To highlight the influence of stationary flows on static MHD equilibria, we neglected compressibility effects and used the assumption of field-aligned, incompressible stationary flows. These assumptions imply an analogy between magnetic field and velocity field as well as an analogy between MHS and incompressible SMHD: The assumption of incompressibility allows us to transform magnetostatic equilibria into stationary ones by using a non-canonical transformation. We find that the occurrence of flow driven current sheets is closely related to the gradient of the plasma flow or, to be precise, to the gradient of the Alfvén Mach number perpendicular to the 

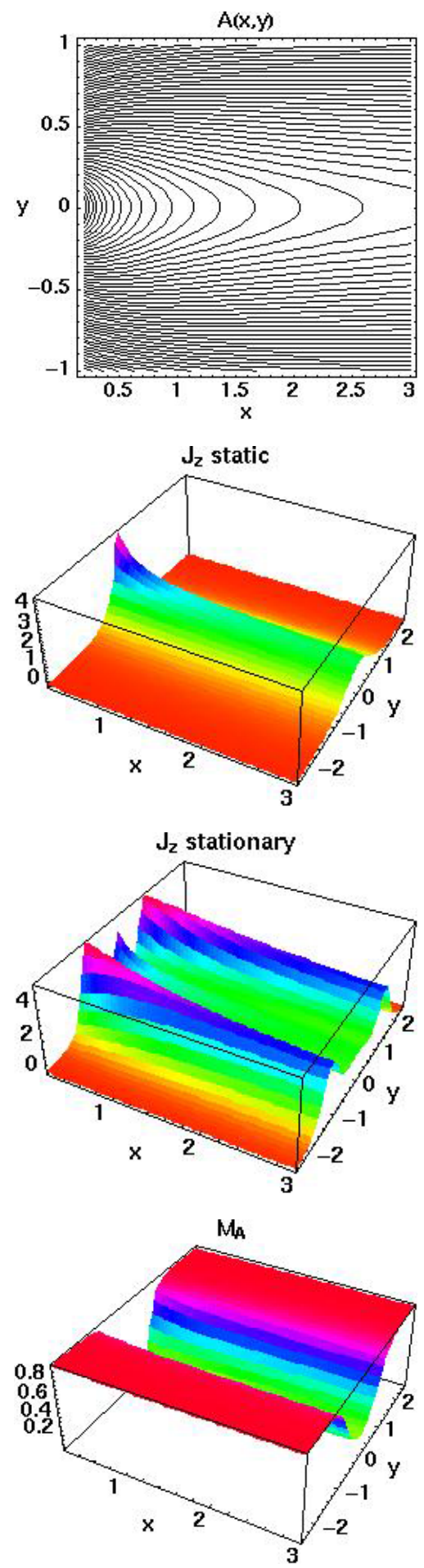

Fig. 6. Same transformation formula as Fig. 2, but for a nonlinear current in the form $J_{z}(A) \propto \exp (-c A)$. For the transformation we used $M_{1}=0.0, M_{2}=0.95$ and $d=2$. For a better visualization we show the negative current $-j_{z}(x, y)$ in the second and third panel. magnetic field lines. Along the field lines the Mach number is always constant for incompressible stationary flows. As the gradients in the Alfvén Mach number can be very large, because the typical length scale of the flow is smaller than the length scale of the magnetic field, the occurence of current sheets is correlated with the appearance of vortex sheets. Such configurations can be closely connected to local breakdowns of the frozen-in-flux theorem, or shortly to magnetic reconnection (Eyink and Aluie, 2006). Magnetic reconnection in turn plays a major role in eruptive space plasma processes like magnetospheric substorms or solar flares.

In principle it is possible to compare our theoretical investigations on the relation between plasma flows and current sheets with observations, in particular in the magnetosphere where in-situ measurements are available. One possibility is using magnetic field and particle data from the CLUSTER-mission, which are carried out simultaneously with four spacecraft. Such multi-spacecraft measurements (with distances between the spacecraft in the range of about $50-10000 \mathrm{~km}$ ) of the magnetic field allow also the estimation of electric currents. By taking moments of the particle data it is possible to compute plasma quantities like density, pressure and the plasma flow velocity. These combined measurements allow at least to estimate gradients in the plasma flow and the thickness of current sheets. A limitation is that structures smaller than the distance of the Cluster-spacecraft cannot be spatially resolved, which implies that flow gradients could be steeper and the current sheets thinner as computed from the measurements. A comparison of data with our model, which relates flows and flow gradients to current sheets, will allow to investigate how consistent different areas in the magnetosphere can be described under the assumption of stationary incompressible MHD.

Acknowledgements. D.H.N. acknowledges financial support from GAAV ČR under grant number IAA300030804. D.H.N. is also grateful to the Max-Planck-Institut für Sonnensystemforschung for the financial support during his visits. The work of T.W. was supported by DLR-grant 50 OC 0501.

The service charges for this open access publication have been covered by the Max Planck Society.

Topical Editor R. Forsyth thanks one anonymous referee for her/his help in evaluating this paper.

\section{References}

Bandle, C.: Existence theorems, qualitative results and a priori bounds for a class of nonlinear Dirichlet problems, Archive for Rational Mechanics and Analysis, 58, 219-238, doi:10.1007/ BF00280742, 1975.

Baranov, V. B. and Krasnobaev, K. V.: On a model of interaction between solar wind and interstellar medium., Kosmicheskie Issledovaniia, 9, 620-622, 1971. 
Baranov, V. B., Krasnobaev, K. V., and Kulikovskii, A. G.: A model of the interaction between solar wind and interstellar medium., Akademiia Nauk SSSR Doklady, 194, 41-44, 1970.

Becker, U., Neukirch, T., and Schindler, K.: On the quasistatic development of thin current sheets in magnetotail-like magnetic fields, J. Geophys. Res., 106, 3811-3826, doi:10.1029/ 2000JA900141, 2001.

Birn, J.: Stretched three-dimensional plasma equilibria with fieldaligned flow, Physics of Fluids B, 3, 479-484, doi:10.1063/1. 859891, 1991.

Birn, J. and Hesse, M.: Reconnection in substorms and solar flares: analogies and differences, Ann. Geophys., 27, 10671078, doi:10.5194/angeo-27-1067-2009, 2009.

Birn, J., Goldstein, H., and Schindler, K.: A theory of the onset of solar eruptive processes, Solar Phys., 57, 81-101, doi:10.1007/ BF00152046, 1978.

Eyink, G. L. and Aluie, H.: The breakdown of Alfvén's theorem in ideal plasma flows: Necessary conditions and physical conjectures, Physica D, 223, 82-92, doi:10.1016/j.physd.2006.08.009, 2006.

Gebhardt, U. and Kiessling, M.: The structure of ideal magnetohydrodynamics with incompressible steady flow, Physics of Fluids B, 4, 1689-1701, doi:10.1063/1.860078, 1992.

Hameiri, E.: Variational principles for equilibrium states with plasma flow, Physics of Plasmas, 5, 3270-3281, doi:10.1063/1. 872995, 1998.

Harris, E. G.: On a plasma sheath separating regions of oppositely directed magnetic fields, Nuovo Cimento, 23, 115-121, 1962.

Harrison, M. G. and Neukirch, T.: One-Dimensional VlasovMaxwell Equilibrium for the Force-Free Harris Sheet, Phys. Rev. Lett., 102, 135003, doi:10.1103/PhysRevLett.102.135003, 2009.

Hood, A. W. and Anzer, U.: A model for quiescent solar prominences with normal polarity, Solar Physics, 126, 117-133, doi: 10.1007/BF00158302, 1990.

Liouville, C.: Sur l'equation aux derivee partilles..., J. de Math., 18, 71-72, 1853 .

Nickeler, D. H., Goedbloed, J. P., and Fahr, H.: Stationary fieldaligned MHD flows at astropauses and in astrotails. Principles of a counterflow configuration between a stellar wind and its interstellar medium wind, aap, 454, 797-810, doi:10.1051/ 0004-6361:20053062, 2006.
Petrie, G. J. D.: Filamentary Loop Systems and Spontaneous Current Sheets in the Solar Corona, The Astrophysical Journal Supplement Series, 166, 378-409, doi:10.1086/505624, 2006.

Petrie, G. J. D. and Neukirch, T.: Self-consistent Three-dimensional Steady-state Solutions of the MHD Equations with Field-aligned Incompressible Flow, Geophys. Astrophys. Fluid Dynam., 91, 269-302, 1999.

Petrie, G. J. D., Vlahakis, N., and Tsinganos, K.: Systematic construction of exact 2-D MHD equilibria with steady, compressible flow in Cartesian geometry and uniform gravity, A\&A, 382, 1081-1092, doi:10.1051/0004-6361:20011650, 2002.

Petrie, G. J. D., Tsinganos, K., and Neukirch, T.: Steady 2D prominence-like solutions of the MHD equations with fieldaligned compressible flow, A\&A, 429, 1081-1092, doi:10.1051/ 0004-6361:20041706, 2005.

Schindler, K.: Physics of Space Plasma Activity, Cambridge University Press, doi:10.2277/0521858976, 2006.

Schindler, K. and Birn, J.: Self-consistent theory of time-dependent convection in the earth's magnetotail, J. Geophys. Res., 87, 2263-2275, doi:10.1029/JA087iA04p02263, 1982.

Schindler, K. and Birn, J.: On the cause of thin current sheets in the near-Earth magnetotail and their possible significance for magnetospheric substorms, J. Geophys. Res., 98, 15477, doi: 10.1029/93JA01047, 1993.

Schindler, K. and Birn, J.: MHD stability of magnetotail equilibria including a background pressure, J, Geophys, Res, (Space Physics), 109, 10208, doi:10.1029/2004JA010537, 2004.

Schmid-Burgk, J.: Finite Amplitude Density Variations in a SelfGravitating Isothermal Gas Layer, ApJ, 149, 727, doi:10.1086/ 149305, 1967.

Wiegelmann, T.: Solar Magnetic Fields: Triple Arcade Structures, Physica Scripta, T74, 77-81, 1998.

Wiegelmann, T. and Schindler, K.: Formation of thin current sheets in a quasistatic magnetotail model, Geophys. Res. Lett., 22, 2057-2060, 1995.

Wiegelmann, T., Schindler, K., and Neukirch, T.: Helmet Streamers with Triple Structures: Weakly Two-Dimensional Stationary States, Solar Phys., 180, 439-460, 1998. 